\title{
Information on doctor and pharmacy shopping for opioids adds little to the identification of presumptive opioid abuse disorders in health insurance claims data
}

This article was published in the following Dove Press journal:

Substance Abuse and Rehabilitation

\author{
Alexander M Walker (D) \\ Lisa B Weatherby' \\ $M$ Soledad Cepeda ${ }^{2}$ \\ Daniel C Bradford ${ }^{3}$
}

'World Health Information Science Consultants, Dedham, MA, USA; ${ }^{2}$ Janssen Research and Development, Titusville, NJ, USA; ${ }^{3}$ Advanced Analytics, IQVIA, Plymouth Meeting, PA, USA
Correspondence: Alexander M Walker World Health Information Science Consultants, 3 Allied Drive, Dedham, MA 02026, USA

$\mathrm{Tel}+\mathrm{I} 78 \mathrm{I} 7427280$

Email Alec.Walker@WHISCON.com
Background: Doctor and pharmacy shopping ("Shopping") for opioids is related to opioid abuse and is associated with opioid overdose and death. Lacking identifiers for prescribers and pharmacies, many data resources (notably the US FDA's Sentinel System) cannot evaluate Shopping. We used data in which presumptive Shopping could be identified. We investigated whether US health insurance claims data could perform as well as Shopping to identify people with evidence for opioid abuse.

Methods: In this cross-sectional study, we examined health insurance claims from 164,923 persons with at least two dispensing of opioids in 18 months, the first occurring in 2012 . Evidence for the presence of a possible opioid abuse disorder was drawn from predictive patterns of drug fills, diagnoses and care-seeking identified in a companion research project, and Shopping was determined using a published index. The prevalence of presumptive opioid abuse was examined across levels of Shopping. The comparison between Shopping and insurance-claims-derived covariates in the detection of apparent opioid abuse was examined in multiple regression analyses.

Results: Despite a strong correlation between presumptive opioid abuse and Shopping, most persons with extensive Shopping did not manifest presumptive opioid abuse, and half of the population with presumptive opioid abuse did not exhibit Shopping. As Shopping ranged from "None" to "Extensive," the prevalence of presumptive opioid abuse increased from 0.28 to 5.0 per 100 . The discriminating power of Shopping for identifying opioid abuse could be replaced using insurance claims data.

Conclusion: The results suggest that patient characteristics that can be inferred from insurance claims data provide as complete discrimination of persons with presumptive opioid abuse as does a full assessment of doctor and pharmacy shopping. The inference rests on patterns of health services and drug dispensing that are indicative of doctor-pharmacy shopping and of opioid abuse. There was no direct evaluation of patients. The extent to which the conclusions are generalizable beyond the study population - Americans with health insurance coverage in the early part of this decade - is uncertain in a quantitative sense. The qualitative conclusion is that diagnostic data in health insurance databases can be predictive of behaviors consistent with opioid abuse and that more elaborate indices such as doctor and pharmacy shopping may add little.

Registration: ClinicalTrials.gov study number: NCT02668549.

Keywords: doctor and pharmacy shopping, opioids, abuse, screening, prediction

\section{Introduction}

Patients' use of multiple prescribers and pharmacies to obtain opioids predicts opioid overdose and death. ${ }^{1-4}$ Diagnoses of opioid abuse in health insurance claims moreover 
are more frequent in persons whose dispensed opioids come from larger numbers of prescribers and dispensing pharmacies. $^{5-7}$ The US Food and Drug Administration (FDA) has required manufacturers of extended-release and long-acting (ER/LA) opioids to undertake a group of studies to quantify and evaluate the risks. ${ }^{8}$ One of those studies, reported here, was to address the further question of whether doctor and pharmacy "shopping" to obtain opioids is a measure of and possibly a proxy for opioid abuse.

The evaluation of shopping requires identification of prescribers and pharmacies, information that is not always readily available. For example, the FDA's Sentinel System of over 200 million persons does not carry identifiers for prescribers or dispensing pharmacy outlets. ${ }^{9}$ Opioid abuse is also difficult to identify clearly in insurance data. The best current algorithms are plausible but complex indices derived from patterns of medical care utilization and drugs dispensing. (See "Presumptive opioid abuse" below.)

Unlike data maintained by US regulatory authorities, the availability of pharmacy and prescriber identifiers in the data used for in this report does permit the assessment of Shopping in relation to presumptive opioid abuse in a large and fully enumerated US population. Like the US regulatory data, the information employed here also incorporates complete insurance claims records, and we have had the opportunity to implement the best current measures of presumptive opioid abuse. The objective of the present analysis was to assess the extent to which the added information on the identities of doctors and pharmacy outlets could be useful for detecting presumptive opioid abuse. We find that the simpler and more accessible measures perform as well as Shopping in separating out presumptive opioid abuse.

\section{Methods}

The study is a period cross-sectional analysis undertaken using deidentified data derived from United States health insurance claims files and drug dispensing information. The period cross-sectional design refers to the use of patient characteristics that are derived from the accumulated experience over a defined observation period, which in this case was 18 months. The analysis is a cross-tabulation and modeling of characteristics built up over the 18 months of observation.

The study was registered with ClincialTrials.gov (Study number: NCT02668549). The protocol and statistical analysis plan for this work were reviewed by the FDA and modified in response to the FDA's requests before any analyses were undertaken. The full protocol is available with the
Supplementary Materials. The source data were analyzed under license with the medical informatics firm IQVIA (formerly known as Quintiles-IMS), which can provide access to other parties on similar terms. The present report is a condensation of material presented to the FDA and reflects comments from that agency.

\section{Data sources}

Data were obtained from IQVIA for the years 2012 through 2014. All patient identifiers had been encrypted before the addition of any patient information to the IQVIA data warehouse. Consistent but encrypted identifiers (so-called "tokens") mean that individual data from the various databases can be merged without use of protected health information. Use of the IQVIA data does not require ethical or institutional review board approval.

\section{LRx}

The IQVIA Longitudinal Prescription ("LRx") database as used for this study had information on 234 million patients and in 2013 covered $86 \%$ of all retail dispensing in the US. LRx data are assembled and continuously updated to facilitate one of IQVIA's business lines as a clearinghouse for drugs payment information. LRx includes prescription fill data from pharmacy chains, food stores, mass merchandisers, independent stores and long-term care facilities across the US. From each of the outlets, the database captured all fills, both in person and through the mail, both paid by insurance and paid entirely by the consumer. LRx distinguishes unique pharmacies, unique prescribers and unique medical practices and can capture records generated by patients who filled prescriptions in more than one state. ("Practices" are professionally affiliated groups of prescribers. Practice information was available for about $45 \%$ of prescribers represented in LRx in 2013.)

\section{PharMetrics Plus}

The IQVIA PharMetrics Plus database holds pharmacy, provider and facility claims for patients enrolled in many US health plans. Through 2012, the starting year for the present analysis, PharMetrics Plus included data on a cumulative total of approximately 75 million persons. Ninetyseven percent of the patients was commercially insured, $2 \%$ of the patients were insured by Medicare (US Federal insurance for the elderly), and $1 \%$ by Medicaid (US Federal/state insurance for the poor). The health plans included in PharMetrics Plus have a wide geographic US representation. PharMetrics Plus also contains hospital 
discharge data and demographic information. About 54\% of the members enrolled in plans contributing data to PharMetrics Plus in 2012 had mental health coverage. All data are cleaned, linked by patient and formatted to facilitate population research.

\section{Linkage}

There were approximately 36 million patients whose records could be linked between the LRx and PharMetrics Plus databases for this study, using the common patient tokens available in each data source. The linked number represents $48 \%$ of the 75 million patients in PharMetrics Plus and $15 \%$ of the 234 million patients in LRx.

\section{Study population}

IQVIA prepared files for the study population by crossreferencing and extracting records from the data sources described above. The study population consisted of adult patients with data recorded in the PharMetrics Plus database for whom a link could be established with IQVIA LRx and who had either an immediate-release/short-acting (IR/SA) or an ER/LA opioid dispensing in 2012 plus another opioid dispensing within 18 months (548 days). The patients were limited to those with mental health coverage who had at least 12 months of observation in LRx before and 18 months after their first opioid dispensing in 2012, unless it appeared that their follow-up had been truncated by death. Patients with presumed death, as evidenced by a termination of coverage following care for a possibly terminal event, were included up to the date of termination of coverage.

The steps that IQVIA followed to create the study population were as follows:

1. Identify all persons with a fill for an opioid prescription in 2012 in the LRx database. Restrict the pull to persons whose opioid fills were from pharmacy outlets that appeared in LRx consistently for the entire study period (12 months prior through 18 months following first dispensing in 2012).

2. Among those identified in (1), find persons who also had records in PharMetrics Plus.

3. Restrict the population in (2) to persons aged 18 years and older at the time of the first opioid fill in 2012.
4. Restrict the population in (3) to those whose entry in LRx (first fill of any prescription) was at least 365 days before the first opioid fill in 2012 .

5. Restrict the population in (4) to those with a second opioid fill within 18 months following first fill in 2012.

6. Restrict the population in (5) to those for whom at least one PharMetrics fill had also appeared LRx. Duplicate fill records for the same product, dose and quantity on the same day to the same person were treated as a single dispensing in both PharMetrics and LRx.

7. Restrict the population in (6) to persons who did not have any long-term care activity during the period from first opioid fill in 2012 through the next 18 months. Long-term care included nursing homes, rest homes, convalescent homes and institutional providers, all not associated with hospitals. The long-term care exclusion also included persons with services from nursing home providers, visiting nurses and home health care providers.

8. Restrict the population in (7) to persons with either a. PharMetrics membership extending continuously at least 18 months after the date of first opioid or diuretic dispensing in 2012,

b. A presumed death.

9. Restrict analysis to persons for whom LRx captured $100 \%$ of PharMetrics Plus fills for drugs other than opioids during the 18 months following first opioid or diuretic dispensing in 2012.

10. Restrict analysis to persons with mental health coverage.

11. Restrict analysis to those whose age and gender agreed between LRx and PharMetrics Plus.

The counts of included individuals at the steps leading to the final population are given in Table 1 .

\section{Doctor and pharmacy shopping}

The term shopping as used in this study refers to dispensing of opioids from prescriptions (1) from multiple providers who were not in shared medical practices and (2) dispensed at different pharmacy outlets. Previously, we identified a measure of doctor and pharmacy shopping for opioids. ${ }^{10}$ Simple counts for each patient of dispensing that derived from different pharmacies and different prescribers proved the best discriminator of possible aberrant behavior in opioid recipients as compared to a control 
Table I Steps in selection of study population

\begin{tabular}{|l|r|c|}
\hline Step & Count & $\begin{array}{c}\text { Percent } \\
\text { retention }\end{array}$ \\
\hline I. Patients with dispensing of an opioid & $39,921,629$ & \\
during 2012 in LRx & & \\
2. Patients in PharMetrics Plus & $4,227,917$ & 10.6 \\
3. Age $\geq 18$ years & $4,031,423$ & 95.4 \\
4. Prior history of 365 days in LRx & $2,766,388$ & 68.6 \\
5. Have a second fill in 548 days & $1,539,560$ & 55.7 \\
6. Any PharMetrics Plus dispensings in LRx & 642,365 & 41.7 \\
7. No long-term care activity & 639,884 & 99.6 \\
8. Enrollment through 548 days or pre- & 392,879 & 61.2 \\
sumed death & & \\
(Presumed death) & $(3823)$ & \\
9. LRx captured I00\% of PharMetrics Plus & 181,002 & 46.1 \\
I0. Mental health coverage & 168,187 & 92.9 \\
II. Age and gender agree across LRx and & 164,923 & 98.1 \\
PharMetrics Plus & & \\
\hline
\end{tabular}

Abbreviations: LRx-IQVIA, longitudinal prescription database; PharMetrics PlusIQVIA, pharmetrics plus insurance claims database.

group of persons filling prescriptions for diuretics. The categories are defined by the number of prescriber practice sites from which opioid prescriptions were obtained in the 18-month observation period and the number of pharmacy outlets that filled the prescriptions (Table 2).

The multilevel entity with the categories defined in Table 2 is referred to as "Shopping" throughout this report.

\section{Presumptive opioid abuse}

Investigators at Kaiser-Permanente Washington have created and assessed an algorithm that uses elements available in US health insurance data to identify presumptive opioid abuse (Manuscript in preparation. D. Carrell, personal communication, July 2019). The algorithm development began with the medical records of a sample consisting of 2000 adults who had received at least 60 days' supply of ER/LA opioids over a 90-day period and who had at least 36 months of continuous

Table 2 Definitions of levels of doctor and pharmacy shopping over 18 months of follow-up

\begin{tabular}{|c|c|}
\hline Level & Definition \\
\hline None & (No contributory dispensing) OR ( 2 practices and 2 outlets) \\
\hline Minimal & $\begin{array}{l}\text { ( } 2 \text { practices AND }>2 \text { outlets) OR }(2 \text { outlets AND } \\
>2 \text { practices) }\end{array}$ \\
\hline Moderate & $\begin{array}{l}\text { ( } 3 \text { practices AND } \geq 3 \text { outlets) OR ( } 4 \text { practices AND } \\
\text { ( } 3 \text { or } 4 \text { outlets) OR ( } 5 \text { practices and } 3 \text { outlets) }\end{array}$ \\
\hline Extensive & $\begin{array}{l}\text { ( } 4 \text { practices AND } \geq 5 \text { outlets) OR ( } 5 \text { practices AND } \\
\geq 4 \text { outlets) OR ( } \geq 6 \text { practices AND } \geq 3 \text { outlets) }\end{array}$ \\
\hline
\end{tabular}

representation in the Kaiser-Permanente electronic medical record. The sampled individuals had received a median 1208 days' supply of ER/LA medications. Abstracters used a standardized review protocol to classify records for evidence of opioid abuse. After identification of evidence for opioid abuse in the medical record, the next step was to systematically explore 1122 potential predictors of opioid abuse. The variables evaluated as predictors had been proposed by clinicians on the study team, who suggested diagnoses, treatments and patterns of care that were likely to indicate the presence of opioid abuse. Statistical identification of predictors was accomplished through LASSO regression, a variant on logistic regression that revises the coefficients of weak predictors to zero, thereby permitting statistically stable estimates of the effects of remaining predictors. The final product of the development process was a score for each person in the study population. We made minor adaptations to the Kaiser-Permanente algorithm to fit the available insurance data and time frame of the study. These are noted in Table A1 in the Supplementary Materials, which gives the definitions of the 52 predictors along with the corresponding weights that were used to derive the score for presumptive opioid abuse.

To reduce the effect of erroneous classification of persons as having opioid abuse, we chose a cut-point of the Kaiser-Permanente score with a high specificity $(90 \%)$. The sensitivity for chart-inferred opioid abuse was just over $50 \%$. In this report, we refer to cases identified using the Kaiser-Permanent rules and the chosen cut-point as "presumptive opioid abuse." The sensitivity figure means that one should expect that about half of the individuals whose medical records would be classed as having evidence for opioid abuse on expert review were missed. The fact that the score consisted entirely of behaviors and medical responses associated with abuse meant that the included instances were those with the largest number of behaviors and diagnoses and were therefore likely to be those that were most clear-cut.

The data used in the presumptive opioid abuse algorithm do not include counts of prescribing physicians or dispensing pharmacies, so there is no logical redundancy between the definitions of shopping and of presumptive opioid abuse. As noted in the next section, we also excluded from consideration any of the insurance claims characteristics that were near-restatements of the elements that went into the score for opioid abuse. 


\section{Other personal and dispensing characteristics}

A broad range of personal, medical and drug dispensing characteristics can be inferred from insurance claims data, and we started with those laid out in our previous publication reporting on the development and assessment of the Shopping measure. ${ }^{10}$ The characteristics fell into broad categories of demographics, characteristics of opioids and concomitant medications, and diagnoses. Demographics (age, sex and region) were drawn from the contract files in PharMetrics Plus. Drug dispensing characteristics were based on the insurance claims and the LRx files, which gave date, substance, dose, form and quantity for every dispensing. Diagnoses were drawn from diagnoses associated with insurance claims and were placed in categories that were prespecified by the FDA or that appear as chapters of the International Classification of Diseases $9^{\text {th }}$ Revision coding system. From a longer list of potential predictors, we removed any that were simply restatements or likely close correlates of the characteristics of the opioid abuse score presented in the Supplementary Materials (Table A1). We used backward elimination in a logistic regression with presumptive opioid abuse as the dependent variable to reduce the list of potential covariates. Supplementary Materials Table B1 lists all the retained characteristics, together with their associated ORs for opioid abuse, both with and without inclusion of Shopping in a logistic model (see the section "Data analyses"). Additionally, we examined the total morphine milligram equivalents (MMEs) of opioid dispensed. ${ }^{11,12}$

\section{Data analyses}

The period prevalence of presumptive opioid abuse (the fraction of the study population that met criteria during the
18 months of observation) was tabulated by category of Shopping (Table 2) and analyzed in a logistic regression model with presumptive opioid abuse as the dependent variable and Shopping as a categorical predictor. The overall dependence of presumptive opioid abuse on Shopping category was evaluated in a test for trend across the ordered categories of Shopping. The regression was repeated with covariates for personal characteristics, and the $\mathrm{C}$-statistics for discrimination between persons with and without presumptive opioid abuse were calculated for Shopping alone, for covariates alone and for Shopping and covariates together, to check independent contributions of each. The relative prevalence of presumptive opioid abuse in categories of Shopping is also presented graphically with statistical adjustment for personal characteristics inferred from the insurance claims and for MME dispensed in the 18 months of observation.

\section{Results}

\section{Shopping in relation to presumed opioid abuse}

The overall 18-month period prevalence of presumptive opioid abuse was 0.48 per 100 persons, based on 793 identified cases of presumptive opioid abuse among 164,923 study subjects. Prevalence rose across levels of Shopping, up to 5.04 per 100 among persons with "Extensive" Shopping (Table 2).

Nearly half of presumptive opioid abuse cases (49.3\%; 391 of 793) occurred among persons with no identified Shopping and $72.4 \%$ were among those at the two lowest Shopping levels, "None" and "Minimal." Only 129 of the 793 cases of presumptive opioid abuse (16.3\%) were found in the "Extensive" Shopping category. The great majority $(95.0 \%)$ of people with "Extensive" Shopping

Table 3 The distribution of the study population and of presumptive opioid abuse cases over categories of doctor and pharmacy shopping

\begin{tabular}{|c|c|c|c|c|}
\hline $\begin{array}{l}\text { Shopping } \\
\text { category }\end{array}$ & Population (\%) & $\begin{array}{l}\text { Presumptive opioid } \\
\text { abuse }\end{array}$ & $\begin{array}{l}\text { Prevalence of presumptive opioid abuse per } \\
100 \text { persons } \\
(95 \% \mathrm{Cl})\end{array}$ & $\begin{array}{l}\text { OR }^{a} \\
(95 \% \mathrm{CI})\end{array}$ \\
\hline None & I39,977 (84.9) & 391 & $0.28(0.25,0.3 \mathrm{I})$ & Ref. \\
\hline Minimal & $|6,43|(10.0)$ & 183 & I.II $(0.96,1.29)$ & $4.02(3.37,4.80)$ \\
\hline Moderate & $5956(3.6)$ & 90 & $1.51(1.23,1.85)$ & $5.48(4.35,6.90)$ \\
\hline Extensive & $2559(1.6)$ & 129 & $5.04(4.26,5.96)$ & $18.95(15.47,23.22)$ \\
\hline Total & $164,923(100.0)$ & 793 & $0.48(0.45,0.52)$ & \\
\hline
\end{tabular}

Notes: ${ }^{a}$ ORs and $\mathrm{Cls}$ derived from logistic regression with Shopping categories predicting presumptive opioid abuse. Chi-square 3 d.f. $=1500.52$, $p<0.00 \mathrm{I}$. Test for trend $z=35.42, p<0.001$. C-statistic $=0.689$.

Abbreviation: Shopping, doctor and pharmacy shopping. 
did not demonstrate presumptive opioid abuse. The dichotomy between a strong directional association and weak explanatory power is highlighted in the summary statistics given in Table 3. The test for the trend of increasing prevalence of opioid abuse in relation to higher levels of Shopping was highly significant $(p<0.0001)$, while in the same data the C-statistic was 0.689 , indicating that the discrimination between presumptive opioid abuse and noncases was modest.

Table $\mathrm{C} 1$ in the Supplementary Materials describes the period prevalence over 18 months of each of the patient characteristics in categories of Shopping and according to the presence of presumptive opioid abuse. Opioid abuse varies dramatically across many of the categories of patient characteristics, but it varies much less across categories of Shopping within characteristics. In the tabulations of Table $\mathrm{C} 1$, the characteristics that were most associated with the higher prevalence of presumptive opioid abuse are: dispensing of antidepressants, antipsychotics and hypnotics; ER/LA opioids; opioid dispensing against self-payment (as opposed to payment by insurance) and total MME dispensed.

\section{Multivariable analyses}

The joint contributions of the predictors were modeled in the multivariable analyses. With mutual adjustment among the variables retained for the analysis, the patient characteristics that were the strongest predictors of designation as presumptive opioid abuse were census region of residence (New England highest by approximately two-fold), 20 or more dispensing of hypnotics (3.3-fold increase for 20+ vs no dispensing), any dispensing of psychostimulants (approximately 2-fold increase) and any opioid dispensing paid for by the patient rather than the insurer (3.3-fold increase). The fact of having received more than 2500 MMEs in the 18 months of observation increased by more than five-fold a patient's odds of designation as presumptive opioid abuse.

The coefficients for Shopping estimated from the logistic regressions with and without covariate adjustment are illustrated in Figure 1 as ORs. Figure 1 presents the ORs for levels of Shopping, (1) with no adjustments, (2) after adjustment only for the patient characteristics and (3) after adjustment for the patient characteristics plus total MME dispensed.

Figure 1 indicates that Shopping taken by itself is strongly associated with presumptive opioid abuse, but that essentially all the association is accounted for by the association between Shopping and data readily identifiable from insurance claims. For example, the most important single reduction in the apparent marginal impact of Shopping came with the addition of MME to the logistic

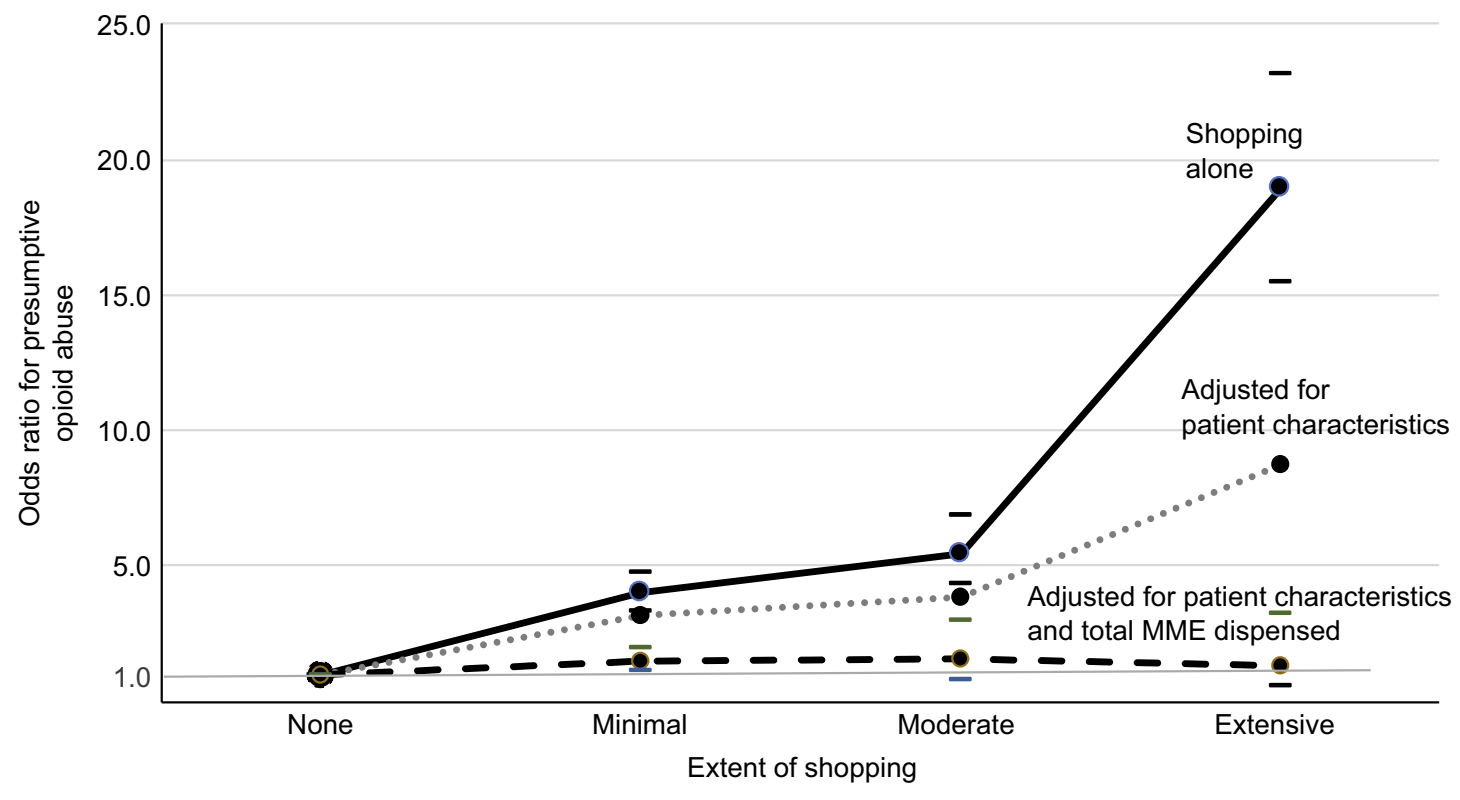

Figure I ORs linking presumptive opioid abuse to doctor and pharmacy shopping, alone and with adjustment for information available in insurance claims data. Notes: Horizontal line segments above and below the points for "Shopping alone" and "Adjusted for patient characteristics and total MME dispensed" denote $95 \%$ confidence bounds for the ORestimates. An ORof I.0 indicates no association between Shopping and presumptive opioid abuse.

Abbreviations: Shopping, doctor and pharmacy shopping; MME, morphine milligram equivalents of opioids dispensed during 18 months. 
regression model. C-statistics for discrimination of presumptive opioid abuse cases from noncases, shown in the footnote to Figure 1, indicate that substantial improvement in discrimination resulted from inclusion of patient characteristics (0.797 vs 0.689 for Shopping alone) and patient characteristics and MME together $(0.813$ vs 0.689$)$ in the regression equation.

\section{Discussion}

Presumptive opioid abuse, as measured here, was better predicted by insurance claims-recorded diagnoses and drugs and by characteristics of opioid dispensing than by apparent shopping behavior. The great majority of persons (2430/2559) with extensive Shopping did not meet the criteria for presumptive opioid abuse, most of which were present in persons whose Shopping was classed as "none" or "minimal" (574/793).

Our earlier work showed that extensive shopping represents a use of prescribers and pharmacies that is far outside of the norm of patient conduct. ${ }^{10}$ By their behavior (if they consume the opioids dispensed to them), people with extensive Shopping would seem to suffer from some disorder related to opioid use. Still, 95 of 100 persons with extensive Shopping did not meet insurance claims criteria for presumptive opioid abuse. An important alternative possibility, which we did not investigate here, is that Shopping is an indicator of drug diversion, which is common and has been identified as an important component of opioid-related deaths. ${ }^{1,13}$

The opioid epidemic in the United States has evolved rapidly since the era covered by this report. Many persons whose opioid use disorders began with prescription drugs now are commonly dependent on illicit drugs instead. ${ }^{14}$ Illicit opioids themselves have become a frequent entry point into opioid abuse. ${ }^{15}$ Death rates from the combination of cocaine and psychostimulants along with opioids are rising rapidly. ${ }^{16}$ Many poly-substance opioid-related deaths appear to be among persons who do not have access to commercial health insurance and who are therefore not represented in the populations studied here. ${ }^{17}$ The changes mean that the findings here need to be considered as a snapshot of US data in 2012-2014.

The current widespread access to illicit drugs in the United States means that strategies such as Shopping to obtain drug may have become a smaller part of national patterns of opioid abuse than they were in the years of the study and in early years when Shopping was widely investigated as an indicator of potential abuse..$^{3,7,18-21}$ The availability of illicit drugs of abuse also undermines the rationale for algorithms for detecting abuse that utilize dispensing information for prescribed drugs. Recourse to validated, direct patient assessments may be necessary. The cost of doing large numbers of such assessments in the general population suggests that risk-based sampling may be required, as was used in a recent development of an algorithm to extract drug overdose events from health insurance data. $^{22}$

Estimates of the prevalence of opioid abuse in the US general population vary widely and depend on the ascertainment method. Our results fall in the range of earlier results for the same calendar era. Roland et al summarized studies using ICD codes in insurance database from 2010 to 2014 to find prevalence estimates among opioid users from $0.2 \%$ to $1.1 \% .^{23}$ At the other extreme of sensitivity, and using data from a few years later, Mojtabi et al reviewed data from the US National Survey on Drug Use and Health to find that $4.4 \%$ of US adults who had received an opioid product in 2015 and 2016 endorsed the statement that they had used it "in any way a doctor did not direct." 24

On the opioid abuse score, we chose a cutoff point with relatively low sensitivity in order to achieve high specificity and high predictive value. That is, we wanted to be sure that the cases presumptively identified would be true cases. In this, we appear to have been successful. The very low prevalence of presumptive opioid abuse among persons whose level of Shopping was "None" means specificity of the algorithm for presumptive opioid abuse in the present setting must have been $>99 \%$. The absolute levels of risk reported here are underestimated by the algorithm, but none of the qualitative conclusions of this study are affected.

The current findings are drawn from the claims files of commercial US health insurers contributing to the PharMetrics Plus database from 2012 through 2014. Persons with commercial health insurance in the US during the years of the study were largely Americans with nongovernmental employment and their dependents. The present results are very probably generalizable to that class of persons. There is a strong correspondence between the medical and demographic characteristic of persons with different levels of Shopping in the present data and those identified in work on other populations in the US, including persons receiving Medicaid and persons whose experience is captured in prescription drug monitoring programs. ${ }^{1}$ The correspondence supports the idea that the 
findings may be applicable to insured persons and perhaps US residents more generally.

Multiplicity of prescribers and of dispensing pharmacies has been integral to many prior indices and predictive equations for risk for abuse and/or addiction., ${ }^{2,5,7,8}$ The present findings suggest that such indices may point to persons at higher risk, but at the same time they miss most cases of opioid abuse.

The most important limitations to the present work lie in the specificity of the research venue and the absence of review of the underlying medical record, with a consequent reliance on a complex algorithm for defining opioid abuse, developed in a different patient population. While we have tried to limit our direct inference to the performance of measures in insurance databases, a natural extension that we cannot directly address would be to large electronic medical record databases. The reliance on chart review from previous studies leaves open the question as to whether chart review here would have produced similar results. The study period encompasses the height of prescription opioid dispensing in the US. The applicability of the results to the current situation, in which nonprescription opioids have taken on a major role in the US epidemic of opioid abuse, is necessarily a matter of speculation.

\section{Conclusion}

Doctor and pharmacy shopping for opioids is a presumably aberrant behavior that here and elsewhere has been shown to be correlated with opioid abuse. The statistical correlation notwithstanding, a quantified measure of Shopping is a poor absolute predictor of opioid abuse, and many persons with presumptive opioid abuse have no evidence for doctor and pharmacy shopping at all. Simpler measures of patient characteristics including use of other psychoactive agents and male gender are approximately as useful as Shopping for indicating persons with opioid abuse, but even collectively these do no provide clinically actionable indicators for opioid abuse.

\section{Acknowledgments}

This study was funded by the Opioid PMR Consortium (OPC), which is comprised of companies that hold NDAs of extended-release/long-acting analgesics, working in response to collective post-marketing requirements from the US Food and Drug Administration (http://www.fda.
gov/Drugs/DrugSafety/InformationbyDrugClass/ ucm363722.htm). This study is part of a program of 11 post-marketing study requirements, as required by the FDA, being implemented by the OPC. The study was designed in collaboration between OPC members and World Health Information Science Consultants (WHISCON) with input from FDA. WHISCON maintained intellectual freedom in terms of publishing final results. The data on which this analysis is based were prepared by Stephanie Roy, Heather Preston, Yingli Yuan, Michel Denarie and R. Goksu of IQVIA.

\section{Disclosure}

AMW and LBW are employees of WHISCON. DCB is an employee of IQVIA. MSC is an employee of Janssen Research and Development. AMW holds stocks in Pfizer Inc. AMW and LBW report funding from Opioid PMR Consortium, outside of the submitted work, during the conduct of the study. MSC holds stocks in Janssen R\&D. The authors report no other conflicts of interest in this work.

\section{References}

1. Hall AJ, Logan JE, Toblin RL, et al. Patterns of abuse among unintentional pharmaceutical overdose fatalities. JAMA. 2008;300 (22):2613-2620. doi:10.1001/jama.2008.802

2. Paulozzi LJ, Kilbourne EM, Shah NG, et al. A history of being prescribed controlled substances and risk of drug overdose death. Pain Med. 2012;13(1):87-95. doi:10.1111/j.1526-4637.2011.01260.x

3. Peirce GL, Smith MJ, Abate MA, Halverson J. Doctor and pharmacy shopping for controlled substances. Med Care. 2012;50(6):494-500. doi:10.1097/MLR.0b013e31824ebd81

4. Gwira Baumblatt JA, Wiedeman C, Dunn JR, Schaffner W, Paulozzi LJ, Jones TF. High-risk use by patients prescribed opioids for pain and its role in overdose deaths. JAMA Intern Med. 2014;174(5):796-801. doi:10.1001/jamainternmed.2013.12711

5. White AG, Birnbaum HG, Schiller M, Tang J, Katz NP. Analytic models to identify patients at risk for prescription opioid abuse. $\mathrm{Am}$ J Manag Care. 2009;15(12):897-906.

6. Sullivan MD, Edlund MJ, Fan M-Y, Devries A, Brennan Braden J, Martin BC. Risks for possible and probable opioid misuse among recipients of chronic opioid therapy in commercial and medicaid insurance plans: the TROUP study. Pain. 2010;150:332-339. doi:10.1016/j.pain.2010.05.020

7. Cepeda MS, Fife D, Kihm MA, Mastrogiovanni G, Yuan Y. Comparison of the risks of shopping behavior and opioid abuse between tapentadol and oxycodone and association of shopping behavior and opioid abuse. Clin J Pain. 2014;30(12):1051-1056. doi:10.1097/AJP.0000000000000067

8. US Food and Drug Administration. New safety measures announced for extended-release and long-acting opioids. Updated November 5, 2017. Available from: http://www.fda.gov/Drugs/DrugSafety/ InformationbyDrugClass/ucm363722.htm, Accessed August 11, 2017

9. US Food and Drug Administration. Sentinel common data model. Available from: https://www.sentinelinitiative.org/sentinel/data/distrib uted-database-common-data-model/sentinel-common-data-model. Accessed May 5, 2018 
10. Walker AM, Weatherby LB, Cepeda MS, Bradford D, Yuan Y. Possible opioid shopping and its correlates. Clin J Pain. 2017;33 (11):976-982. doi:10.1097/AJP.0000000000000483

11. Pereira J, Lawlor P, Vigano A, Dorgan M, Bruera E. Equianalgesic dose ratios for opioids. a critical review and proposals for longterm dosing. J Pain Symptom Manage. 2001;22(2):672-687.

12. Carman WJ, Su S, Cook SF, Wurzelmann JI, McAfee A. Coronary heart disease outcomes among chronic opioid and cyclooxygenase-2 users compared with a general population cohort. Pharmacoepidemiol Drug Saf. 2011;20(7):754-762. doi:10.1002/pds.2131

13. Katz NP, Birnbaum HG, Castor A. Volume of prescription opioids used nonmedically in the United States. J Pain Palliat Care Pharmacother. 2010;24(2):141-144. doi:10.3109/15360281003799098

14. Cicero TJ, Ellis MS, Harney J. Shifting patterns of prescription opioid and heroin abuse in the United States. $N$ Engl J Med. 2015;373(18):1789-1790. doi:10.1056/NEJMc1505541

15. Cicero TJ, Ellis MS, Kasper ZA. Increased use of heroin as an initiating opioid of abuse. Addict Behav. 2017;74:63-66. doi:10.1016/j. addbeh.2017.05.030

16. Kariisa M, Scholl L, Wilson N, Seth P, Hoots B. Drug overdose deaths involving cocaine and psychostimulants with abuse potential — United States, 2003-2017. MMWR Morb Mortal Wkly Rep. 2019;68:388-395. 10.15585/mmwr.mm6817a3external icon. doi:10.15585/mmwr.mm6 $805 \mathrm{a} 4$

17. Barocas JA, Wang J, Marshall BDL, et al. Sociodemographic factors and social determinants associated with toxicology confirmed polysubstance opioid-related deaths. Drug Alcohol Depend. 2019;8(200):59-63. doi:10.1016/j.drugalcdep.2019.0 3.014
18. Katz N, Panas L, Kim M, et al. Usefulness of prescription monitoring programs for surveillance-analysis of Schedule II opioid prescription data in Massachusetts, 19962006. Pharmacoepidemiol Drug Saf. 2010;19(2):115-123. doi:10.1002/pds.1878

19. Cepeda MS, Fife D, Chow W, Mastrogiovanni G, Henderson SC. Assessing opioid shopping behaviour: a large cohort study from a medication dispensing database in the US. Drug Saf. 2012;35 (4):325-334. doi:10.2165/11596600-000000000-00000

20. Cepeda MS, Fife D, Chow W, Mastrogiovanni G, Henderson SC. Opioid shopping behavior: how often, how soon, which drugs, and what payment method. J Clin Pharmacol. 2013;53(1):112-117. doi: $10.1177 / 0091270012436561$

21. Chilcoat HD, Coplan PM, Harikrishnan V, Alexander L. Decreased diversion by doctor-shopping for a reformulated extended release oxycodone product (OxyContin). Drug Alcohol Depend. 2016;1(165):221-228. doi:10.1016/j.drugalcdep.2016.0 6.009

22. Green CA, Perrin NA, Hazelhurst B, et al. Identifying and classifying opioid-related overdoses: a validation study. Pharmacoepidemiol Drug Saf. 2019; doi:10.1002/pds.4772. Epub ahead of print.

23. Roland CL, Lake J, Oderda GM. Prevalence of prescription opioid abuse/abuse as determined by international classification of diseases codes: a systematic review. $J$ Pain Palliat Care Pharmacother. 2016;30(4):258-268. doi:10.1080/15360288.2016. 1231739

24. Mojtabai R, Amin-Esmaeili M, Nejat E, Olfson M. Abuse of prescribed opioids in the United States. Pharmacoepidemiol Drug Saf. 2019;28(3):345-353. doi:10.1002/pds.4743
Substance Abuse and Rehabilitation

\section{Publish your work in this journal}

Substance Abuse and Rehabilitation is an international, peerreviewed, open access journal publishing original research, case reports, editorials, reviews and commentaries on all areas of addiction and substance abuse and options for treatment and rehabilitation. The manuscript management system is completely online and includes a very quick and fair peer-review system. Visit http://www.dovepress. com/testimonials.php to read real quotes from published authors. 